\title{
Oesophageal Squamous Dysplasia
}

\author{
Michael Auld ${ }^{1}$ (D) $\cdot$ Havish Srinath ${ }^{1} \cdot$ Eshwarshanker Jeyarajan $^{2}$ \\ Published online: 28 May 2018 \\ (C) Springer Science+Business Media, LLC, part of Springer Nature 2018
}

\section{Epidemiology and Aetiology}

Squamous cell carcinoma (SCC) of the oesophagus is the most common neoplasia of the oesophagus worldwide [1]. In Australia, the incidence of oesophageal SCC is approximately 1 in 100,000 with males having a higher predilection [2]. The diagnosis is predominantly through endoscopic examination of the oesophagus. The overall incidence of oesophageal SCC has been trending downward over the last several decades $[3,4]$. Western countries, in particular, have lower rates with some South African and Middle Eastern countries experiencing rates exceeding 100 in 100,000 [3]. Males are diagnosed with SCC in a ratio upwards of eight to one [4]. It represents a significant disease burden, associated with substantial morbidity, quality of life and a poor prognosis. Oesophageal cancer is graded by the TNM system which divides the carcinoma based on the depth of invasion through mucosa, submucosa and into adjacent structures [5].

The development of squamous dysplasia is a multifactorial process with many risk factors contributing to the chronic inflammation and carcinogen intake. Smoking and ethanol consumption are by far the most common risk factors for this process, with synergistic effects [2]. The decline in cigarette use in developed countries is believed to contribute to the decrease in oesophageal SCC [2]. Another important carcinogen thought to be involved in the development of squamous dysplasia is nitrosamines. This compound and its precursors are commonly found in high concentrations in pickled vegetables, smoked fish and cured meat accounting for some of the geographical difference in incidence where these foods are

This manuscript has not been published or under consideration elsewhere.

Michael Auld

Michael.auld@uqconnect.edu.au

1 General Surgery Department, Ipswich Hospital, Ipswich Hospital, Chelmsford Avenue, Ipswich, QLD 4305, Australia

2 General Surgery Department, Gold Coast University Hospital, Southport, QLD, Australia consumed regularly [3]. Several genetic differences in oesophageal SCC occur in Asian populations compared to Caucasians which may contribute to the higher incidence identified [6]. Variances in enzyme functionality for alcohol dehydrogenase, particularly amongst the Japanese population, may also contribute [7]. Numerous medical conditions predispose patients to the development of chronic oesophageal inflammation, increasing their risk. Achalasia, Plummer-Vinson syndrome and scleroderma all increase the risk of oesophageal squamous dysplasia by oesophageal stasis [3]. An association between oesophageal carcinoma with another head and neck cancer has been well described, often secondary to smoking as the carcinogen [8]. A further disease that increases the risk of head and neck cancers is Fanconi anaemia representing a rare inherited genetic defect $[9,10]$. The association between head and neck SCC and human papillomavirus has been well established; however, an aetiologic link with oesophageal SCC has not been identified $[11,12]$. Patients who have previously ingested a caustic agent or been exposed to radiation therapy to the chest also have increased the risk of developing oesophageal cancer due to the direct mutagenic effects of these agents. Some studies have suggested that consuming very hot beverages (greater than 70 degrees Celsius), low socioeconomic status and malnutrition also have interplay in increasing the risk of oesophageal SCC [3].

\section{Squamous Cell Carcinoma Versus Adenocarcinoma}

Oesophageal SCC and adenocarcinoma represent different pathological conditions with different pathophysiology, risk factors, locations, prognosis and treatment [13]. SCC develops through squamous hyperplasia, low-grade intraepithelial neoplasia to high grade, and carcinoma in situ before becoming invasive. The progressive accumulation of dysregulation in cell cycle regulators including TP53, retinoblastoma-associated protein $(R B)$ and cyclin-dependent kinase inhibitor $2 \mathrm{~A}$ $(C D K N 2 A)$ is a prominent feature even in precursor lesions 
[14]. Adenocarcinoma, in comparison, exhibits a progression through oesophagitis, Barrett's metaplasia and dysplasia often secondary to gastro-oesophageal reflux disease. The repeated DNA injury characteristically causes increasing base-pair transversions in the mutational profile. Two further steps are proposed with loss of tumour suppressor genes (CDKN2A and TP53) before chromosomal instability occurs, especially copynumber alterations [15]. Although Barrett's metaplasia responds to proton pump inhibitor therapy, there is no evidence that this also aids prevention of squamous dysplasia progressing to SCC [15]. Adenocarcinoma is found below the level of the tracheal bifurcation in $95 \%$ of cases compared to SCC which more often occurs superior to the bifurcation [4]. Prognostically, the difference is significant with SCC having a 10 -year survival of approximately $20 \%$ compared to adenocarcinoma which has above 40\% [9]. The difference has further been recognised with the most recent American Joint Committee on Cancer (AJCC) oesophageal malignancy staging that now differentiates between SCC and adenocarcinoma [5]. Different centres have suggested that given the good response to neoadjuvant therapy, squamous cell carcinomas may, potentially, have a better prognosis $[4,16]$.

\section{Management}

Oesophageal SCC, once metastatic, has a poor prognosis and treatment available is limited to palliative chemoradiation and stenting. This is often the case when symptoms develop [3]. However, if detected earlier, there are options to endoscopically or surgically manage the dysplasia or carcinoma (see Table 1). Therefore, initial staging scans with or without endoscopic ultrasound are important for adequate identification of nodal disease. When low-grade, high-grade or carcinoma in situ is detected, referral for endoscopic mucosal resection or radiofrequency ablation has both been shown to have efficacious results in terms of disease-free progression $[17,18]$.
Surveillance would normally continue at 3 monthly intervals to assess for clearance post treatment. Once the lesion has become invasive, endoscopic options are only available until it invades submucosa (T1b), except in certain centres specialised in this treatment. Treatment for invasive oesophageal SCC not amenable to endoscopic management is either oesophagectomy, if comorbidities permit, or definitive chemoradiotherapy [4]. If surgery is feasible, 5-year survival rates with no neoadjuvant treatment are reported as $12-27 \%$ [4]. This can be increased to $57 \%$ with neoadjuvant treatment. Immunotherapies are under investigation with several studies targeting tyrosine-kinase receptors and epidermal growth factor receptors with limited success [15]. In particular, the Cancer Oesophagus Gefitinib (COG) trial investigated the effectiveness of gefitinib for oesophageal cancer after chemotherapy with no improvement in overall survival [19].

\section{Surveillance}

Endoscopy remains the gold standard in the detection of pre-malignant and malignant changes of the oesophagus [20]. Developing techniques for the detection of early oesophageal squamous dysplasia are under investigation including non-endoscopic cytosponge and balloon sampling. Early patchy changes of dysplasia can be missed on conventional white light endoscopy so the development of biomarkers (i.e. Trefoil Factor 3) from cytosponge specimens may be possible [21]. Identifying which population groups to screen with endoscopy remains contentious. Several patient populations as described previously bear mention for surveillance as they confer an increased risk of malignancy. In patients with an oropharyngeal cancer or primary oesophageal malignancy, there is an increased risk of another upper gastrointestinal malignancy. Some research has advocated for surveillance at 6 monthly intervals; however, a cost-benefit analysis or survival benefit
Table 1 Oesophageal squamous dysplasia and invasive carcinoma surveillance and management

\begin{tabular}{ll}
\hline Endoscopic and staging findings & Management \\
\hline Low-grade dysplasia & Early repeat endoscopy \pm Lugols iodine staining with repeat biopsy \\
High-grade dysplasia & $\begin{array}{c}\text { Referral for endoscopic management including radiofrequency } \\
\text { ablation and endoscopic mucosal resection }\end{array}$ \\
Tis (carcinoma in situ) & Referral for endoscopic management including radiofrequency ablation \\
& and endoscopic mucosal resection, post resection surveillance often \\
& 3 months \\
T1a (T1b in certain centres) & Refer for endoscopic submucosal dissection or mucosal resection -or- \\
& Refer for surgical resection \\
T2 & Referral for surgical resection \\
T3-T4, N1-N3 & Chemoradiation as definitive treatment or neoadjuvant prior to surgical \\
& resection in selected cases \\
\hline
\end{tabular}


has not been proven for this $[1,3,6]$. Both achalasia and Plummer-Vinson syndrome confer an increased risk; however, there is no strong data to support routine surveillance $[3,8]$. If these patients have additional risk factors or symptoms, further screening may be necessary. Evidence does exist for the surveillance of patients post caustic ingestion, with endoscopy starting at approximately 15 years after the insult. Following the initial endoscopy, surveillance intervals of 1-3 years have been recommended $[1,22]$. Tylosis, a rare genetic condition predisposing to hyperkeratosis and oesophageal cancer, has also been advocated for surveillance every $1-3$ years, beginning from the age of 30 onwards [1]. As can be shown from these varied time frames and evidence, no consensus exists for many conditions that predispose to oesophageal malignancy. Higher risk populations should raise concern when red flags, such as dysphagia or weight loss, develop to prompt further investigation [23].

\section{Clinical Practice Points}

- Great variation exists between Barrett's oesophagus and oesophageal squamous dysplasia in terms of risk factors and pathology.

- Alcohol and tobacco are the most common risk factors for oesophageal squamous dysplasia and carcinoma. Cessation of both is vital to preventing disease progression.

- Red flag symptoms such as dysphagia, unexplained weight loss or a family history of oesophageal squamous cancer may be the initial prompt for endoscopic evaluation.

- Surveillance is necessary in certain high-risk populations including tylosis and prior caustic agent ingestion.

\section{Future Direction}

Given that oesophageal malignancies have such poor outcomes, it is the focus of intense investigation for new immunotherapies and biomarkers. The KEYNOTE-028 study used pembrolizumab, an anti-PD1 (programmed cell death protein 1) agent, with $29 \%$ of oesophageal SCC responding to therapy [24]. A further promising agent under investigation in the phase III CHECKMATE 032 trial was nivolumab [25]. This checkpoint inhibitor showed promise with responses of $27 \%$ for PD1-positive disease and improved 1-year survival [25]. More research into these agents may yield an increased survival benefit over current therapies.

\section{Summary}

Oesophageal malignancy can be a challenge to diagnose given its vague symptomatology and late presentation. Several highrisk factors and populations may raise red flags for further investigation. The outcomes are generally poor once metastatic; however, newer techniques for the endoscopic treatment of in situ disease are available. Surveillance intervals, although controversial, should be aimed at detecting disease progression, especially in the high-risk population.

Author Contribution All authors are in approval with this manuscript. Each author had significant input in this article in terms of the conceptualisation, drafting, content and final approval.

\section{Compliance with Ethical Standards}

Conflict of Interest The authors declare that they have no conflict of interest.

\section{References}

1. Evans J, Early D, Chandraskhara V, et al. The role of endoscopy in the assessment and treatment of oesophageal cancer. Gastrointest Endosc. 2013;77:328-34.

2. Arnold M, Soerjomataram I, Ferlay J, Forman D. Global incidence of oesophageal cancer by histological subtype in 2012. Gut. 2015;64:381-7.

3. Messman H. Squamous cell cancer of the oesophagus. Best Pract Res Clin Gastroenterol. 2001;15:249-65.

4. Yong E, Han XP, Watson D, et al. Outcome following surgery for squamous cell carcinoma of the oesophagus. ANZJSurg. 2009;79: 724-8.

5. Rice T, Patil D, Blackstone E. 8th edition AJCC/UICC staging of cancers of the esophagus and esophagogastric junction: application to clinical practice. Ann Cardiothoracic Surg. 2017;6:119-30.

6. Chen S, Zhou K, Yang L, Ding G, Li H. Racial differences in esophageal squamous cell carcinoma: incidence and molecular features. Biomed Res Int. 2017;2017:1-9.

7. Deng J, Chen H, Zhou D, et al. Comparative genomic analysis of esophageal squamous cell carcinoma between Asian and Caucasian patient populations. Nat Commun. 2017;8:1-9.

8. Scherubl H, Lampe B, Faiss S. Screening for oesophageal neoplasia in patients with head and neck cancer. Br J Cancer. 2002;86:239-43.

9. Alsobahi F, Collis S, Hunter K. The role of Fanconi Anaemia pathway in sporadic non-FA associated head and neck squamous cell carcinoma. Eur J Cancer. 2016;61:S35.

10. Tipples K, Raouf S. Treatment of oesophageal squamous cell carcinoma in a patient with Fanconi anaemia. Clin Oncol. 2008;3:383-4.

11. Bass A, Laird P, Shmulevich I. Integrated genomic characterization of oesophageal carcinoma. Nature. 2017;12:169-75.

12. Petrick J, Wyss A, Butler A. Prevalence of human papillomavirus among oesophageal squamous cell carcinoma cases: systematic review and meta-analysis. Br J Cancer. 2014;110:2369-77.

13. Siewert J, Ott K. Are squamous and adenocarcinomas of the oesophagus the same disease? Semin Radiat Oncol. 2007;17:38-44.

14. Liu X, Zhang M, Ying S, Zhang C, Lin R, Zheng J, et al. Genetic alterations in esophageal tissues from squamous dysplasia to carcinoma. Gastroenterology. 2017;153:166-77. 
15. Smyth E, Lagergren J, Fitzgerald R, Lordick F, Shah M, Lagergren P, et al. Oesophageal cancer. Nature. 2017;3:1-21.

16. Siewert J, Stein H, Feith M, et al. Histologic tumor type is an independent prognostic parameter in esophageal cancer: lessons from more than 1,000 consecutive resections at a single center in the Western World. Ann Surg. 2001;234:360-7.

17. Haidry R, Butt M, Dunn J, et al. Radiofrequency ablation for early oesophageal squamous neoplasia: outcomes form United Kingdom registry. World J Gastroenterol. 2013;19:6011-9.

18. Mannath J, Ragunath K. Endoscopic mucosal resection: who and how? Ther Adv Gastroenterol. 2010;4:275-82.

19. Dutton S, Ferry D, Blazeby J, et al. Gefitinib for oesophageal cancer progressing after chemotherapy (COG): a phase 3, multicentre, double-blind, placebo-controlled randomised trial. Lancet Oncol. 2014; 15:894-904.

20. Song L, Wilson B. Endoscopic detection of early upper GI cancers. Best Pract Res Clin Gastroenterol. 2005;19:833-56.
21. Lao-Sirieix P, Fitzgerald R. Screening for oesophageal cancer. Nature. 2012;9:278-86.

22. Evans J, Early D, Fukami N. The role of endoscopy in Barretts oesophagus and other premalignant conditions of the esophagus. Gastrointest Endosc. 2012;76:1087-94.

23. Walker M, Harris A, Edwards G, Talley N. A GP primer for understanding upper gastrointestinal tract biopsy reports. AFP. 2015;44: 706-11.

24. Doi T, Piha-Paul SA, Jalal SI, Mai-Dang H, Saraf S, Koshiji M, et al. Updated results for the advanced esophageal carcinoma cohort of the phase Ib KEYNOTE-028 study of pembrolizumab (MK3475). J Clin Oncol. 2016;34(4_suppl):7-7.

25. Kang Y, Satoh T, Ryu M, et al. Nivolumab (ONO-4538/BMS936558) as salvage treatment after second or later-line chemotherapy for advanced gastric or gastro-esophageal junction cancer (AGC): a double-blinded, randomized, phase III trial. J Clin Oncol. 2017;35:2-2. 